OnLine Journal of Biological Sciences 8 (1): 25-31, 2008

ISSN 1608-4217

(C) 2008 Science Publications

\title{
Isolation and Characterization of Novel Metal Accumulating Extracellular Protease Secreting Bacteria from Marine Coastal Region of Digha in West Bengal, India
}

\author{
${ }^{1}$ Susmita Roy, ${ }^{1}$ Kaushal Mishra, ${ }^{1}$ Sanhita Chowdhury, ${ }^{2}$ Ashoke R Thakur and ${ }^{1}$ Shaon Raychaudhuri \\ ${ }^{1}$ Department of Biotechnology, West Bengal University of Technology, BF-142, Sector 1, Salt Lake, \\ Calcutta-700064, India \\ ${ }^{2}$ West Bengal State University, Barasat North 24 Parganas, Barasat Government College \\ Annexe Building, 10KNC Road, Kolkata-00124, India
}

\begin{abstract}
Problem Statement: The objective of the present piece of work was to explore the existing biodiversity of protease secreting microbes in a marine ecosystem, the site selected being the coastal region of West Bengal (Digha), India. Approach: The culture dependent method was used for isolation and characterization of novel microbes from the point of biotechnological application. The isolate obtained was characterized on basis of its morphological, biochemical, physiological properties and finally its molecular identity was revealed through $16 \mathrm{~S}$ rDNA analysis. The complete characterization would ease the application of this isolate for various purposes which would require the understanding of the detailed properties. Results: The isolation was done on milk medium while further characterization was done in Luria Bertani broth as well as Carbon Minimal Salt medium. The isolate was found to be DNase \& protease positive, spore forming, Gram-positive bacilli and exhibited growth in wide range of substrates, temperature $\left[20^{\circ} \mathrm{C}-40^{\circ} \mathrm{C}\right] \& \mathrm{pH}$ [5.5-10]. The Scanning Electron Micrograph revealed a unique pililike bridge between every 3-4 bacterial cells, which indicates the characteristic stacking pattern of growth found in certain groups of bacteria. Moreover it was found to grow in presence of a wide range of metals namely iron, lead, mercury, zinc, aluminum, copper, nickel, cadmium and silver. Energy dispersive X Ray Fluorescence analysis conducted for two of the metals, chromium and copper indicated the intracellular accumulation of these metals. Conclusion: This property provides potential for its application in remediation as well as recovery of heavy metals. The isolate with this property can be a potential candidate to carry out bioaugmentation.
\end{abstract}

Key words: Marine Environment, carbon minimal salt medium, pililike bridge, heavy metals

\section{INTRODUCTION}

Microbes account for more than $90 \%$ of ocean biomass. The marine microbial group including bacteria, archaea, lower eukaryotes \& their associated viruses are found to be diverse as their origin vary-from the ice-covered polar regions of the Arctic \& Antarctic to the boiling hypothermal vents in the depth of the sea. Still much of the microbial life remains unknown, because of the fact that culture dependent identification could not reveal the entire existing microbial diversity. Marine microbes are reported to play important roles in the earth system from being the major primary producers in the ocean and acting as sources of natural products like enzymes and antibiotics.

One of the important contribution of the microbes in aquatic system is in the geochemical cycle of earth and in some way they explain the evolution of living organisms from inorganic matter ${ }^{[1]}$. The microbes inhabiting the aquatic environment do have importance in the population dynamics and thereby contributing to the functional aspect operating in the ecosystem ${ }^{[2]}$. The diverse group of microbes have applications in various fields like production of enzymes, drugs and various other commercial products ${ }^{[3-6]}$. Taking into account these importance, the primary goal would be to explore the marine environment to look for novel microbes which can have various applications.

Today microbes do have importance in the management and sustenance of environment ${ }^{[7]}$. Many reports present the role of microbes in detoxification of contaminated sites, may be by their interaction with heavy metals or degradation of chemicals like halogenated, aromatic compounds ${ }^{[8-15]}$. Not only marine

Corresponding Author: Susmita Roy, Department of Biotechnology, West Bengal University of Technology, BF-142, Sector 1, Salt Lake, Calcutta-700064, India 
environment but microbes from different ecosystems were reported to play significant role in maintaining the ecosystem as well as played varied roles to decontaminate the environment ${ }^{[16]}$.

The present study reports the characterization of one of the isolate from a marine site of West Bengal, India. The basic objective was to screen out potential microbes, to characterize them and to exploit their properties in various applications.

\section{MATERIALS AND METHODS}

Isolation of pure strain: Milk media (10\% milk, $0.3 \%$ yeast extract and $1.5 \%$ agar) was used as a selective medium for isolation of protease secreting microbes, the source being the water collected from coast of West Bengal (Digha). From a mixed population of microbes isolated by spread plate technique, pure isolate was obtained by repeated streak-plate method on Luria Bertani agar medium (LB medium) (1\% tryptone, $0.5 \%$ yeast extract, $0.5 \%$ sodium chloride, $1.5 \%$ agar).

\section{Characterization of the bacteria}

Morphological characterization: Morphological characterization of the isolate was based on the light and electron microscopy. Other than simple and gram staining, the detailed characterization was conducted through various other staining procedures for detection of endospore, capsule, flagella as per standard procedure. The bright field microscopy was conducted at $100 \mathrm{X}$ magnification on a Zeiss Axiostar Plus microscope. Scanning Electron Microscopy (SEM) revealed the ultrastructure of the cell. The bacterial cell fixation and preparation technique for SEM was followed as reported earlier ${ }^{[17]}$.

Biochemical characterization: Biochemical characterization included the test for presence of enzymes namely protease, DNase, lipase, lecithinase, oxidase \& catalase. The specific ready made medium (Himedia laboratories) were used for the enzymatic assay. The detailed procedure of enzymatic assay and result assessment was as reported earlier ${ }^{[17]}$.

Physiological characterization: Physiological characterization includes determination of optimum $\mathrm{pH}$, temperature for growth, efficiency in substrate utilization, growth profile under enriched condition as well as sensitivity for different antibiotics.

- $\quad$ pH profile: $\mathrm{pH}$ is a limiting factor, which governs bacterial growth. To determine the $\mathrm{pH}$ optima, LB medium meant for growth of the isolate was adjusted to different $\mathrm{pH}$ ranging from 2-13 and was seeded with $1 \%$ inoculum. Post overnight growth at $37^{\circ}$ under shaking condition at $150 \mathrm{rpm}$, growth was measured in terms of OD at $660 \mathrm{~nm}$ in Beckman coulter DV- 530 UV Vis spectrophotometer

- Temperature profile: For determination of optimum temperature, $1 \%$ inoculation was provided into LB medium and overnight incubation was done at different temperatures like 4, 10, 20, $30,35,37,40,45$ and $50^{\circ} \mathrm{C}$. The growth was measured in terms of OD at $660 \mathrm{~nm}$ in Beckman coulter DV-530 UV Vis spectrophotometer.

- Substrate profile: The Carbon Minimal Salt medium was supplemented with different organic substrates namely glucose, lactose, maltose, sucrose, starch cellulose, glycerol, fumarate, urea and ethanol. Post inoculation with $1 \%$ overnight culture, incubation was done at $37^{\circ} \mathrm{C}$ under constant shaking at $150 \mathrm{rpm}$ for overnight. Growth was recorded in terms of OD at $660 \mathrm{~nm}$ in a spectrophotometer (Beckman coulter DV-530 UV Vis spectrophotometer).

- Light sensitivity: To determine the lightsensitivity, two sets of LB media were inoculated with $1 \%$ culture, one of the set was incubated in presence of luminescent light and remaining set was incubated in the dark. The temperature and incubation conditions for both were $37^{\circ} \mathrm{C}$ at constant shaking of $150 \mathrm{rpm}$ for overnight. Thereafter growth was checked in terms of OD at $660 \mathrm{~nm}$ in Beckman UV-Vis spectrophotometer.

- Growth pattern: To determine the growth pattern, $1 \%$ of inoculum from overnight grown parent culture was seeded into sterile $100 \mathrm{~mL} \mathrm{LB}$ broth. $\mathrm{OD}$ at $660 \mathrm{~nm}$ was checked in UV-visible spectrophotometer at a gap of $30 \mathrm{~min}$ until the culture reaches stationary phase.

- Antibiotic sensitivity: Antibiotic sensitivity was checked for 18 different antibiotics. The different antibiotics used and the detailed procedure for sensitivity assay was followed as reported by others $^{[17]}$

Metal sensitivity: The tolerance was checked for eleven different heavy metals The metals used in the study and detailed procedure to determine the tolerance profile, in terms of MIC was as per the protocol reported elsewhere ${ }^{[17]}$. The quantitation of metal accumulation was determined by Energy Dispersive X Ray Fluorescence analysis. The protocol for sample preparation and details of the analytical procedure was reported earlier ${ }^{[17,18]}$. 
Pathogenicity detection by real-time PCR: The detection for the presence of pathogenic gene was done using TaqMan kit (Applied biosystems.) for Staphylococcus aureus and Pseudomonas aeruginosa.

Molecular characterization: The molecular characterization was done on the basis of $16 \mathrm{~S}$ rDNA sequence analysis. The steps for the characterization included the genomic DNA isolation followed by PCR amplification of universal $16 \mathrm{~S}$ rDNA gene. The amplified fragment was then cloned into pGEMT easy vector and transformed into $E$ coli DH5 $\alpha$ host. The positive transformants were screened out and sent for sequencing. The details about genomic DNA isolation, the primer sequence, PCR conditions, transformation procedure was as reported by Ray Chaudhuri and Thakur as well as Ray Chaudhuri et al. ${ }^{[19,20]}$.

The sequence obtained was subjected to nucleotide-nucleotide BLAST, a tool for checking percentage of similarity with the existing database. The sequence being novel was submitted to GenBank.

All the experiments were repeated at least thrice and the data represented are the mean.

\section{RESULTS}

Characterization of the isolate: The preliminary characterization was based on morphological and biochemical properties. The isolate was bacilli as demonstrated by light microscopy as well as electron microscopy (Fig. 1, 3). As depicted from staining results presented in Table 1, the isolate was found to be Gram-positive in nature and possessed endospores (Fig. 2). Other structural features like absence of capsule and flagella were clearly put forth by the staining procedures.

The biochemical nature of the isolate indicates presence of enzymes like DNase and protease.

Physiological characterization: The physiological properties of the isolate were concluded in terms of $\mathrm{pH}$, temperature and substrate.

A wide variety of substrates were found to support the growth of the isolate which indicates the metabolic efficiency of the isolate (Fig. 4). The isolate was found to grow within a $\mathrm{pH}$ range of 5-10 with optimum growth at $\mathrm{pH} 7$ (Fig. 5). The temperature range found suitable for growth was between $20-45^{\circ} \mathrm{C}$, optimum being at $35^{\circ} \mathrm{C}$ (Fig. 6). These physiological conditions are reported as the optimum for most of the functions in a living organism.
Growth pattern: The isolate follows a typical sigmoidal pattern of growth curve. An initial lag pase of two hours succeded by five hours of log phase followed by a stationary phase (Fig. 7).

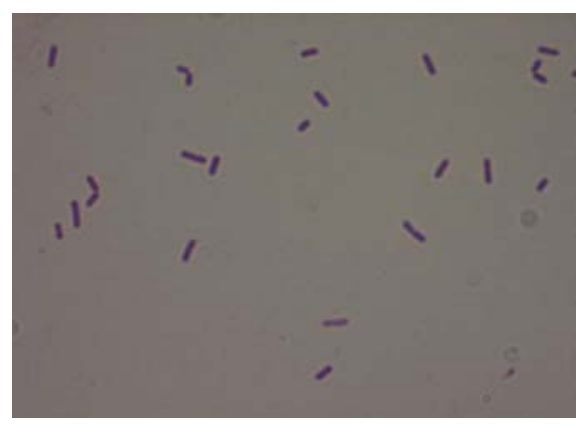

Fig. 1: Light Microscopic photograph at 40X magnification showing Gram-Positive nature of bacillus shaped isolate

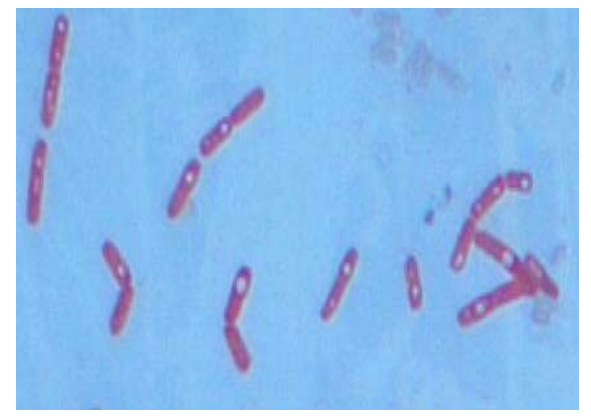

Fig. 2: Light Microscopic photograph (at 100X magnification depicting presence of endospore

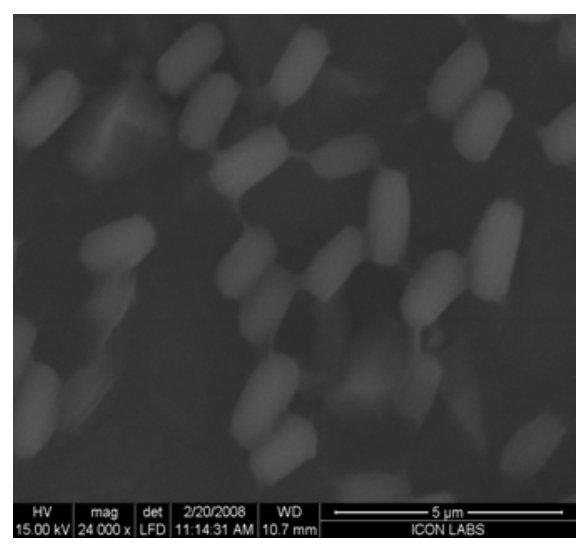

Fig. 3: Scanning Electron Micrograph (9000X) depicting the bacillary shape of the isolate along with pilli like structure found between cells 
Metal sensitivity: The isolate was found to demonstrate tolerance for metals shown in Fig. 8. The minimum tolerance was for metals like mercury, cadmium and silver. The maximum tolerance was demonstrated for iron followed by lead.

Table 1: Table representing the morphological properties based on staining results and biochemical characters based on tests for different enzymes for the isolate. ' + ' indicate the presence of specific morphological character or presence of the enzyme whereas '-' represents absence of the specific feature or property

\begin{tabular}{lllc}
\hline $\begin{array}{l}\text { Morphological characterization } \\
\text { Tests }\end{array}$ & $\begin{array}{l}\text { Biochemical characterization } \\
\text { Test for enzymes }\end{array}$ \\
\hline Simple staining & bacilli & Protease & + \\
Gram-staining & + & DNase & + \\
Spore-staining & + & Catalase & - \\
Capsule staining & - & Oxidase & - \\
Flagella-staining & - & Lecithinase & - \\
Acid-fast staining & - & Lipase & - \\
\hline
\end{tabular}

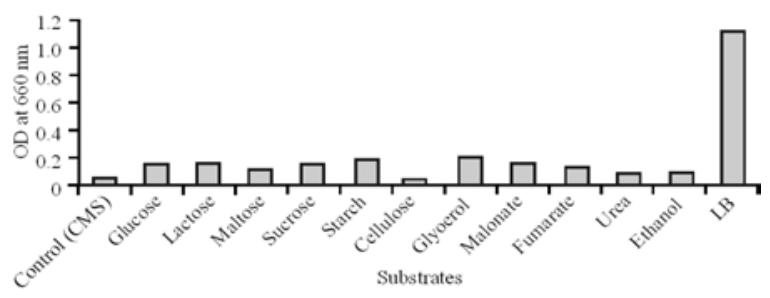

Fig 4: Graph representing the substrate profile of the isolate. Growth was expressed in terms of OD at $660 \mathrm{~nm}$ represented along $\mathrm{Y}$ axis where as the different substrates checked were represented along $\mathrm{X}$ axis. CMS medium without any carbon source was considered as -ve control and Luria Broth medium was taken as +ve control

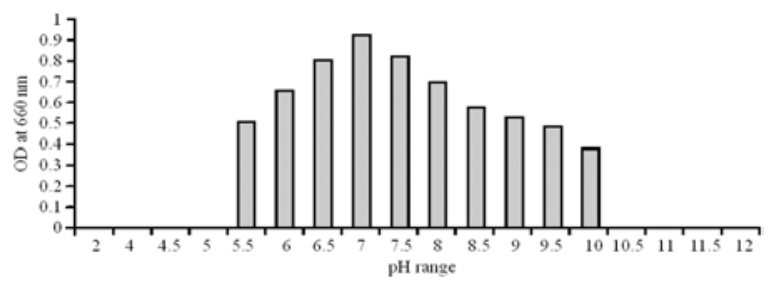

Fig. 5: Bar diagram representing the $\mathrm{pH}$ profile of the isolate. The growth efficiency was represented in terms of OD at $660 \mathrm{~nm}$ plotted along $\mathrm{Y}$ axis with the individual $\mathrm{pH}$ plotted along $\mathrm{X}$ axis

Moreover the EDXRF analysis done for two of the metals among these namely chromium and copper indicates intracellular accumulation of these metals (Table 2).

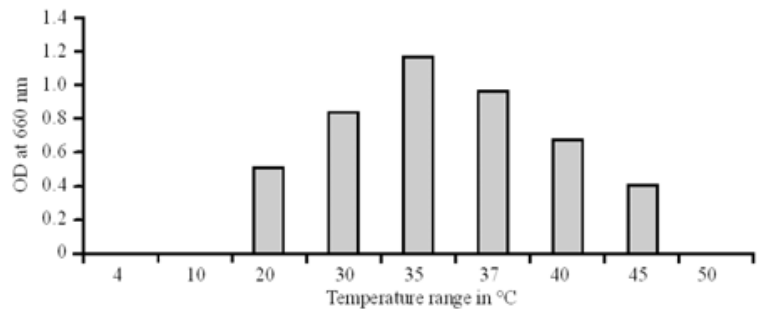

Fig. 6: Graph representing the temperature profile of the isolate. The growth efficiency was represented in terms of OD at $660 \mathrm{~nm}$ plotted along $\mathrm{Y}$ axis and temperature scale was plotted along $\mathrm{X}$ axis

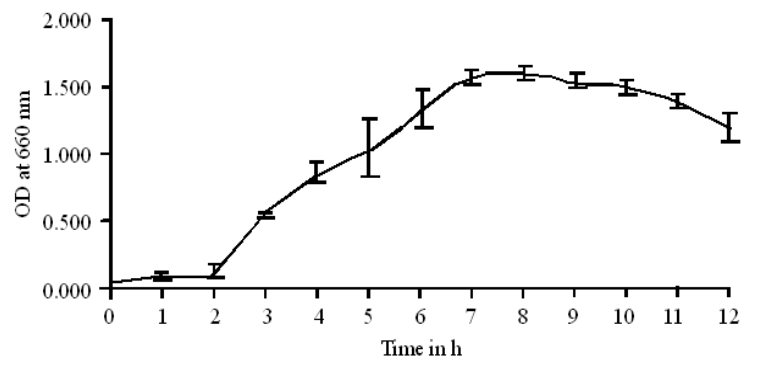

Fig. 7: Graph representing the sigmoid growth pattern of the isolate. The $\mathrm{X}$ axis depicts time in hours where as $\mathrm{Y}$ axis represents bacterial growth presented in terms of OD at $660 \mathrm{~nm}$

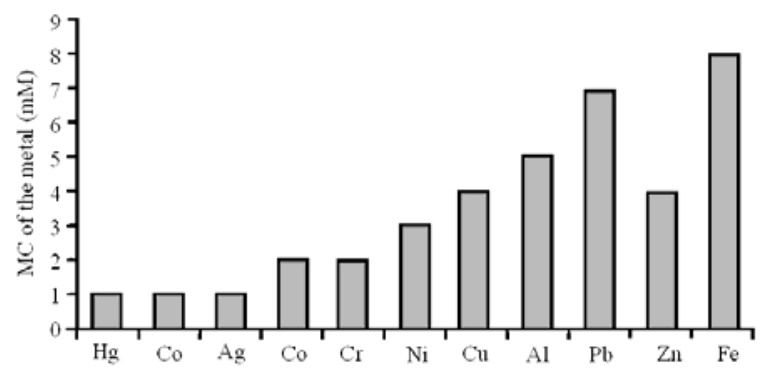

Fig. 8: Graph representing the minimum inhibitory concentration for different metals exhibited by the isolate. The different metals were depicted along the $\mathrm{X}$ axis and the corresponding MIC for specific metals were presented along $\mathrm{Y}$-axis in terms of concentration $(\mathrm{mM})$

Pathogenic Test: The real time PCR based detection for the presence of pathogenic gene indicated negative result for both Staphylococcus aureus and Pseudomonas aeruginosa. Figure 9 depicts the amplification plots obtained using the probes for specific genes for the two strains (Staphylococcus 
aureus and Pseudomonas aeruginosa) mentioned above. As observed from Fig. 9, the internal positive control shows high copy number with respect

Table 2: Table representing the EDXRF data showing the efficiency of metal accumulation by the isolate. The concentration of metal accumulated was concluded from difference of metal concentration between treated and untreated cells. The metal accumulated was presented in terms of ppb

\begin{tabular}{|c|c|c|c|}
\hline \multirow[b]{2}{*}{ Name of metal } & \multicolumn{3}{|c|}{ Metal concentration in ppb } \\
\hline & control & Metal treated cell & accumulation (in ppb) \\
\hline Chromium & 6.834 & 103.2 & 96.366 \\
\hline Copper & 6.136 & 24.26 & 18.124 \\
\hline
\end{tabular}

to $S$. aureus pathogenic gene. Similar results were obtained from the RT-PCR conducted for Pseudomonas (data not shown).

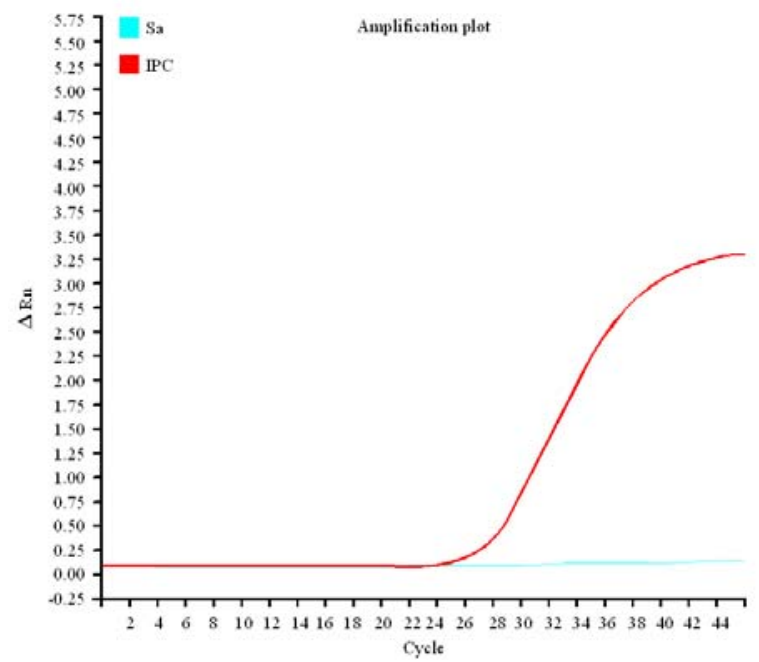

Fig. 9. Amplification plot obtained from real time PCR using specific probes for detection of pathogenic gene in $S$ aureus. $\mathrm{X}$ axis represent the cycle number where as copy no is depicted along $\mathrm{Y}$ axis. Sa represent Staphylococcus aureus pathogenic gene and IPC is internal positive control

Molecular characterization: N-BLAST search of the $16 \mathrm{~S}$ rDNA sequence obtained reveals that the isolate bears maximum similarity with Bacillus cerus (99\%). The sequence obtained being novel was submitted to GenBank and bears the accession no EU-862481.

\section{DISCUSSION}

The presence of enzyme like DNase indicates the possible defense mechanism in bacteria which participate in host prey interaction. This in the long run enables the existence and adaptability of the organism.

The usability of substrates like glucose, lactose, sucrose, fumarate malonate and glycerol indicate towards the aerobic metabolic pathway because these substrates could be easily assimilated and directly enters the Tricarboxylic Acid cycle (TCA). Ethanol not giving substantial growth means that glyoxylate pathway was not supported.

The $\mathrm{pH}$ and temperature profile of an isolate would indicate the adaptability of the isolate to different habitats. These factors do have an effect on survivability since they have roles in enzymatic function as well as overall metabolic efficiency. The isolate being able to withstand wide range of $\mathrm{pH}$ and temperature was capable of surviving in adverse condition.

An idea of the entire growth cycle would facilitate the complete characterzation of the isolate and further enable designing for practical applications.

The metal tolerance data would definitely facilitate their application in remediation of heavy metals. These metals are commonly found in the mine effluents, small scale battery industries, tanneries etc. These tailings could be removed by the application of this isolate. The EDXRF data indicates the potential for the removal of these toxic metals from the site and moreover certain processes can be followed for the recovery of economically important metals from the microbes.

The analysis of partial sequence with $99 \%$ similarity identifies the isolate as Bacillus $s p$.

\section{CONCLUSION}

This preliminary characterization of the isolate regarding its morphological, biochemical and physiological characters as well as the molecular identity would definitely lead to the application of the isolate for various purposes. The property of the isolate for heavy metal uptake would explain the role of this group of microbes in the geochemical mechanism under the water level and it would also facilitate the application of marine microbes for leaching and removal of the toxic metal dumped into the aquatic ecosystem. The reason for non amplification of pathogenic gene in both tests could also be due to its molecular identity which was different from those used for the test. Another important fact regarding this microbe was that in spite of its marine origin it was able to grow and propagate under non halophilic condition. Moreover the protease secreting property of these isolates would open up the exploitation of the marine 
microbes for production of enzymes. Thus the future prospect lies in the application of this isolate for purposes like heavy metal remediation and commercial product development involving enzyme.

\section{ACKNOWLEDGEMENT}

The authors would like to acknowledge the financial support of Department of Atomic Energy, India as well as Department of Biotechnology, India. They would also like to acknowledge the help extended by Dr M Sudarshan of UGC-DAE Consortium for Scientific Research, Calcutta Centre for carrying out the EDXRF analysis. They would thank Bioinormatics Infrastructure Facility (DBT, GOI supported) of West Bengal University of Technology for its computational facility. The authors would acknowledge the help of Ms Madhusmita Mishra during Manuscript preparation.

\section{REFERENCES}

1. Jannasch, H.W. and C.D. Taylor, 1984. Deep sea microbiology. Ann. Rev. Microbiol., 38: 48-514. DOI:10.1146/annurev.mi.38.100184.002415.

2. Evelyn, A., L. Yan, B. Kenneth, W. Phillip, B.J. Grat, 2001. Symbiotic role of marine microbes in living surfaces. Hydrobiologica, 461: 37-40. cat.inist.fr/?aModele $=$ afficheN\&cpsidt $=13418811$

3. Mann, L.B., 2004. Marine Microbiology: Ecology and application. Bio. Scientific. Publishers, ISBN10:1859962882. www.amazon.com/ MarineMicrobiology - Ecology - Applications -Advanced /dp/ 1859962882

4. Kennedy, J., J.R. Marchesi, A.D.W. Dobson, 2008. Marine metagenomics: strategies for the discovery of novel enzymes with biotechnology application from marine environ. Microbial Cell Factories, 7:27 DOI-10.1186/1475-2859-7-27

5. Newman, D.J. and R.T. Hill, 2006. New drugs from marine microbes: The tide is turning. J.Indu. Microbiol. Biotechnol., 33: 539-544. cat.inist.fr/ ?aModele $=$ afficheN\&cpsidt $=17935128$

6. Falkowski, P.G., T. Fenchal, and E.F. Delong, 2008. The microbiological engines that drive earthś biogeochemical cycles. Science, 320: 1034-1039. DOI: $10.1126 /$ science. 1153213.

7. Vidali, M., 2001. Bioremediation An overview. Pure Appl. Chem., 73: 1163-1172. old.iupac.org/publications/pac/2001/pdf/7307x116 3.pdf.
8. Lloyd, J.R., 2002. Bioremediation of metals, the application of micro organisms that make and break minerals. Microbiology, 29: 67-69. www.sgm.ac.uk/pubs/micro_today/pdf/050203.pdf.

9. Gadd, G.M., 2000. Bioremedial potential of microbial mechanisms of metal mobilization and immobilization. Curr. Opinion Biotechnol., 11: 271-279. DOI-10.1016/S0958-1669(00)00095-1

10. Iwamoto, T.M. Nasu, 2001. Current Bioremediation practice and perspective. J. Biosci. Bioeng., $\quad 92$ : 1-8. cat.inist.fr/?aModele $=$ afficheN $\&$ cpsidt $=14131455$.

11. Lloyd, J.R. and D.R. Lovely, 2001. Microbial detoxification of metals and radio nucleotides. Curr. Opinion Biotechnol., 12: 248-253. http://www.sciencedirect.com/science?_ob=Articl eListURL\&_method=list\&_ArticleListID $=804223$ $271 \&$ sort $=\mathrm{d} \&$ view $=\mathrm{c} \& \_$acct $=\mathrm{C} 000050221 \&$ _ver sion $=1 \&$ urlVersion $=0 \&$ userid $=10 \& \mathrm{md} 5=\mathrm{c} 4377$ d40ba $835 \mathrm{~d} 2 \mathrm{f} 84423 \mathrm{c} 035 \mathrm{fcdcb} 3 \mathrm{f}$

12. Leahy, J.G. and R.R. Colwell, 1990. Microbial degradation of hydrocarbons in the environ. Microbiol. Rev., 54: 305-315. 01460749/90/030305-11\$02.00/0.

13. Nicholson, C.A. and B.Z. Fathepure, 2004. Biodegradation of benzene by halophilic and halotolerant bacteria under aerobic conditions. Applied Environ. Microbiol., 1: 1222-1225. DOI: 10.1128/AEM.70.2.1222-1225.2004

14. Debrah, K.T., S. Fukuyama, N. Otonari, F. Taniguchi and M. Ogura, 1999. An inoculum for the aerobic treatment of wastewaters with high concentrations of fats and oils. Bioresour. Technol., 69: 133-139. http://www.sciencedirect.com/science?_ob=Article ListURL\&_method $=$ list\&_ArticleListI $\bar{D}=8042222$ $22 \&$ _sort $=\overline{\mathrm{d}} \&$ view $=\mathrm{c} \&$ acct $=\mathrm{C} 000050221 \&$ versi on $=1 \&$ \& urlVersion $=0 \& \_$userid $=10 \& \mathrm{md} 5=7321 \mathrm{f} 77$ c936f5c449462fa2f7bea99a0 15. Emtiazi, G., H. Shakarami, I. Nahvi and S.H. Mirdamadian, 2005. Utilization of petroleum hydrocarbons by Pseudomonas sp. and transformed Escherichia coli. African J. Biotechnol., $\quad 4$ : 172-176. cat.inist.fr/?aModele $=$ afficheN\&cpsidt $=1743226$

16. Raychaudhuri, S., M. Mishra, P. Nandy and A.R. Thakur, 2008. Waste management: A case study of ongoing traditional practices at east calcutta wetland. Am. J. Agric. Biol. Sci., 3: 315-320. http://www.scipub.org/fulltext/AJAB/AJAB31315 $-320 . p d f$. 
17. Adarsh, V.K., M. Mishra, S. Chowdhury, M. Sudarshan, A.R. Thakur and Ray S. Chaudhuri, 2007. Studies on metal microbe interaction of three bacterial isolates from East Calcutta Wetland. J. Biol. Sci., 7: 80-88. www.scipub.org/fulltext/ojbs/ojbs7280-88.pdf

18. Chowdhury, S., M. Mishra, V.K. Adarsh, A. Mukherjee, A.R. Thakur and S. Ray Chaudhuri, 2008. Novel metal accumulator and protease secretor microbes from east Calcutta wetland. Am. J. Biochem. Biotechnol., 4: 255-264. www.scipub.org/fulltext/ajbb/ajbb43255-264.pdf
19. Raychaudhuri, S. A.R. Thakur, 2006. Microbial genetics mapping of east Calcutta wetland. Curr. Sci., 2: 212-217. www.ias.ac.in/currsci/jul252006/212.pdf

20. Raychaudhuri, S., A.K. Pattanayak and A.R. Thakur, 2006. Microbial DNA extraction from sample of varied origin. Current Sci. 12: 1697-1700.

www.ias.ac.in/currsci/dec252006/1697.pdf 